\title{
Seleção e classificação multivariada de modelos de crescimento não lineares para bovinos Nelore
}

[Selection and multivariate classification of nonlinear growth model for Nelore cattle]

\author{
N.A.M. Silva ${ }^{1}$, A.M.Q. Lana $^{1}$, F.F Silva ${ }^{2}$, F.G. Silveira ${ }^{2}$, J.A.G. Bergmann ${ }^{1}$, \\ M.A. Silva ${ }^{1}$, F.L.B. Toral ${ }^{1}$ \\ ${ }^{1}$ Escola de Veterinária - UFMG \\ Av. Antônio Carlos, 6627 \\ 31270-901 - Belo Horizonte, MG \\ ${ }^{2}$ Universidade Federal de Viçosa - Viçosa, MG
}

\section{RESUMO}

Utilizou-se análise de agrupamento para classificar e selecionar modelos não lineares de crescimento de bovinos Nelore, tendo em vista os resultados de diferentes avaliadores de qualidade de ajuste. Ajustaramse 12 modelos não lineares. A qualidade de ajuste dos modelos foi medida pelo coeficiente de determinação $\left(\mathrm{R}^{2}\right)$, quadrado médio do erro (QME), critério de informação de Akaike (AIC), critério de informação Bayesiano (BIC), erro quadrático médio de predição (MEP) e coeficiente de determinação de predição $\left(\mathrm{R}_{\mathrm{p}}^{2}\right)$. O modelo Brody foi o que apresentou o melhor ajuste para o conjunto de dados.

Palavras-chave: bovino, Nelore, análise multivariada, modelo não linear

\begin{abstract}
This study aimed to evaluate cluster analysis in classifying and selecting non linear models to describe Nelore beef cattle growth based on different goodness of fit criteria tests. A total of 12 non linear models were evaluated based on the following criteria: the determination coefficient $\left(R^{2}\right)$, error mean square (QME), Akaike information criterion (AIC), Bayesian information criterion (BIC), mean quadratic error of prediction (MEP) and predicted determination coefficient $\left(R_{p}^{2}\right)$. The Brody model showed the best adjustment for the data set.
\end{abstract}

Keywords: bovine, Nellore, multivariate analysis, nonlinear model

\section{INTRODUÇÃO}

Atualmente, uma forma prática e consistente de analisar a eficiência produtiva de raças de bovinos de corte é por meio do estudo de curvas de crescimento, pois estas representam uma trajetória longitudinal dos pesos apresentados pelos animais em função do tempo. Modelos de regressão não linear, desenvolvidos empiricamente para relacionar dados de pesoidade, têm se mostrado adequados para descrever curvas de crescimento. Esses modelos apresentam parâmetros com interpretação biológica e, assim, possibilitam maior entendimento do processo de crescimento dos animais. Esses parâmetros são caracterizados principalmente pelo peso à maturidade, que representa o peso na idade adulta, e pela velocidade de crescimento, que representa medida de precocidade do animal (Silva et al., 2010).

Segundo Silveira (2010), na literatura são citados vários modelos não lineares para descrever o crescimento animal, cada um com suas vantagens e desvantagens sob o ponto de vista estatístico. Segundo Oliveira et al. (2000), Mazzini et al. (2005), Mendes et al. (2009) e Silva et al. (2010), os modelos da "família" Richards (Brody, Von Bertalanffy, Richards,

Recebido em 24 de setembro de 2010

Aceito em 25 de março de 2011

E-mail: nataschaalmeida@yahoo.com.br 
Logística e Gompertz) são os mais utilizados para descrever o crescimento de bovinos. Dessa forma, questiona-se sempre qual desses modelos é o mais apropriado para descrever o crescimento corporal de uma população de bovinos. Segundo Silveira et al. (2009), a resposta para tal questionamento pode ser dada mediante informações provenientes de avaliadores da qualidade de ajuste, que permitem indicar estatisticamente o melhor modelo.

Contudo, por causa do grande número de avaliadores de qualidade existentes na literatura, há discordância entre eles para definição do melhor modelo a ser utilizado para descrever o crescimento dos animais. Assim, uma análise que considere simultaneamente todos os avaliadores de qualidade é necessária para estabelecer qual modelo melhor se ajusta aos dados de crescimento. Segundo Silveira (2010), uma proposta interessante seria a utilização de métodos de classificação multivariados, como a análise de agrupamento, ou análise de cluster, a qual permite a formação de grupos homogêneos de acordo com a avaliação simultânea de várias características de interesse.

Uma questão importante em análise de agrupamento refere-se ao critério a ser utilizado para se decidir até que ponto dois elementos do conjunto amostral podem ser considerados como semelhantes ou não. De acordo com Cruz et al. (2004), existem dois tipos de medidas que podem ser consideradas como critérios para a caracterização da semelhança entre os elementos amostrais. São as medidas de similaridade e as de dissimilaridade. Escolhida a medida de semelhança, passa-se, então, a escolher um método de agrupamento. Entre os métodos de agrupamento existentes, os que são mais frequentemente utilizados são os hierárquicos. Porém, uma das grandes lacunas da análise de agrupamento, quando se utilizam métodos hierárquicos, é a determinação do número ótimo de grupos. Sharma (1996) apresenta estatísticas que permitem acessar o número ótimo de grupos, e, dentre estas, destaca-se o índice root mean square standart deviation (RMSSTD), que é de grande utilidade na área de biometria (Cecon et al., 2008).
O objetivo deste trabalho foi utilizar a análise de agrupamento para classificar e indicar o melhor modelo não linear para descrever o crescimento de bovinos Nelore com base em diferentes avaliadores estatísticos de qualidade de ajuste.

\section{MATERIAL E MÉTODOS}

Os dados utilizados são de 410 animais, de ambos os sexos, da raça Nelore, nascidos entre 2001 e 2004, em dois rebanhos da região de Teófilo Otoni, MG. Estes dados fazem parte dos arquivos de registros da Associação Brasileira de Criadores de Zebu, com sede em Uberaba, MG, e referem-se a pesagens trimestrais - do nascimento aos 550 dias -, tendo em vista que elas não ocorriam exatamente a cada três meses. Animais com menos de sete pesagens foram excluídos do estudo.

Um total de 12 modelos de regressão não linear, descritos por Silveira (2010) (Tab. 1), foi ajustado a dados de bovinos Nelore, utilizandose o método dos quadrados mínimos ordinários, cujas soluções foram obtidas por meio do processo iterativo de Gauss-Newton.

Na Tab. 1, o parâmetro $\beta_{1}$ representa o peso adulto, ou peso assintótico do animal, e o parâmetro $\beta_{3}$ a taxa de maturidade, ou velocidade de crescimento, exceto para os dois últimos modelos, Michaelis-Menten Modificado e Weibull, em que a taxa de maturidade é obtida como função dos demais parâmetros. Os modelos que apresentam o parâmetro $\beta_{4}$ possuem ponto de inflexão variável, cuja localização é determinada pelo parâmetro em questão, e os demais modelos ou apresentam o ponto de inflexão fixo, ou não o possuem, como é o caso dos modelos Brody e Michaelis-Menten. De forma geral, não há uma interpretação prática para o parâmetr $\beta$ 2, sendo este considerado como uma constante de integração: $\mathrm{y}_{\mathrm{i}}$ representa a observação da variável dependente designada de variável resposta, e $\mathrm{x}_{\mathrm{i}}$ a variável independente designada de variável explicativa. 
Tabela 1. Modelos de regressão não linear utilizados para descrever curvas de crescimento

$$
\begin{aligned}
& \mathrm{y}_{\mathrm{i}}=\frac{\beta_{1}}{\left(1+\beta_{4} \mathrm{e}^{\left(\beta_{3} \beta_{2}-\mathrm{x}_{\mathrm{i}}\right)}\right)^{\frac{1}{\beta_{4}}}}+\mathrm{e}_{\mathrm{i}} \text { (Schnute) } \\
& \mathrm{y}_{\mathrm{i}}=\beta_{1}-\mathrm{e}^{\left(-\beta_{2}-\beta_{3} \mathrm{x}_{\mathrm{i}}\right)}+\mathrm{e}_{\mathrm{i}} \quad \text { (Meloun II) } \\
& \mathrm{y}_{\mathrm{i}}=\beta_{1}\left(1-\mathrm{e}^{\left(\beta_{3} \beta_{2}-\beta_{3} \mathrm{x}_{\mathrm{i}}\right)}\right)+\mathrm{e}_{\mathrm{i}} \text { (Mitscherlich) } \\
& {\left[\left(\mathrm{y}_{\mathrm{i}}=\left(\beta_{1}-\beta_{2} \mathrm{e}\right)\right]^{-\beta_{3} \mathrm{x}_{\mathrm{i}}}\right)^{3}+\mathrm{e}_{\mathrm{i}} \text { (Brody) }} \\
& \mathrm{y}_{\mathrm{i}}=\frac{\beta_{1}}{\left(1+\mathrm{e}^{\left(\beta_{2}-\beta_{3} \mathrm{x}_{\mathrm{i}}\right)}\right)^{\frac{1}{\beta_{4}}}}+\mathrm{e}_{\mathrm{i}} \\
& y_{i}=\beta_{1} e^{\left.\left(-e^{\left(\beta_{2}-\beta_{3} x_{i}\right.}\right)\right)}+e_{i} \quad \text { (Gompertz) } \\
& \mathrm{y}_{\mathrm{i}}=\frac{\beta_{1} \mathrm{x}_{\mathrm{i}}}{\mathrm{x}_{\mathrm{i}}+\beta_{2}}+\mathrm{e}_{\mathrm{i}} \quad \text { (Michaelis-Menten) } \\
& \mathrm{y}_{\mathrm{i}}=\frac{\beta_{1}}{\left(1+\mathrm{e}^{\left(\beta_{2}-\beta_{3} \mathrm{x}_{\mathrm{i}}\right)}\right)}+\mathrm{e}_{\mathrm{i}} \quad \text { (Logístico) } \\
& \mathrm{y}_{\mathrm{i}}=\frac{\beta_{2} \beta_{3}^{\beta_{4}}+\beta_{1} \mathrm{x}_{\mathrm{i}}^{\beta_{4}}}{\beta_{3}^{\beta_{4}}+\mathrm{x}_{\mathrm{i}}^{\beta_{4}}}+\mathrm{e}_{\mathrm{i}} \text { (Michaelis-Menten modificado) } \\
& \mathrm{y}_{\mathrm{i}}=\beta_{1}-\beta_{2} \mathrm{e}^{\left(-\beta_{3} \mathrm{x}_{\mathrm{i}}\right)}+\mathrm{e}_{\mathrm{i}} \quad(\text { Meloun I }) \\
& y_{i}=\beta_{1}\left(1-\beta_{2} e^{-\beta_{3} x_{i}}\right)^{3}+e_{i} \text { (von Bertalanffy) } \\
& \mathrm{y}_{\mathrm{i}}=\beta_{1}-\beta_{2} \mathrm{e}^{-\mathrm{e}^{\beta_{3}} \mathrm{x}_{\mathrm{i}}^{\beta_{4}}}+\mathrm{e}_{\mathrm{i}}(\text { Weibull })
\end{aligned}
$$

Para avaliar a qualidade de ajuste dos modelos de regressão não lineares, foram utilizados os seguintes avaliadores de qualidade de ajuste: porcentagem de convergência $(\mathrm{C} \%)$, quadrado médio do erro (QME), coeficiente de determinação $\left(\mathrm{R}^{2}\right)$, critério de Akaike (AIC), critério de informação Bayesiano (BIC), erro quadrático médio de predição (MEP) e coeficiente de determinação de predição $\left(\mathrm{R}_{\mathrm{p}}^{2}\right)$ :

$$
\begin{gathered}
\mathrm{QME}=\frac{\sum_{1=1}^{\mathrm{n}}\left(\mathrm{y}_{\mathrm{i}}-\hat{\mathrm{y}}_{\mathrm{i}}\right)^{2}}{(\mathrm{n}-\mathrm{p})}, \\
\mathrm{R}^{2}=1-\frac{\mathrm{SQR}}{\mathrm{SQT}}, \\
\mathrm{AIC}=-2 \operatorname{loglike}+2 \mathrm{p}, \\
\mathrm{BIC}=-2 \log \operatorname{like}+\operatorname{pln}(\mathrm{n}), \\
\mathrm{MEP}=\frac{\sum_{1=1}^{\mathrm{n}}\left(\mathrm{y}_{\mathrm{i}}-\hat{\mathrm{y}}_{\mathrm{i}}^{*}\right)}{(\mathrm{n})}
\end{gathered}
$$

$$
\mathrm{R}_{\mathrm{p}}^{2}=1-\frac{\mathrm{nMEP}}{\sum_{1=1}^{\mathrm{n}} \hat{\mathrm{y}}_{\mathrm{i}}^{* 2}-\mathrm{n}^{2}}
$$

em que: n é o número de observações utilizadas para ajustar a curva, p é o número de parâmetros na função,ŷ i representa os valores estimados, SQR é a soma de quadrados do resíduo, SQT a soma de quadrados total, loglike é o valor do logaritmo da função de verossimilhança considerando-se as estimativas dos parâmetros, $\hat{\mathrm{y}}_{\mathrm{i}}^{*}$ representa os dados estimados considerandose valores dos parâmetros provenientes de um ajuste sem a presença da observação i, e $\bar{y}$ é a média dos n valores.

Após a obtenção dos avaliadores apresentados para cada um dos modelos estudados, confeccionou-se um conjunto de dados multivariados, no qual os modelos correspondem às unidades a serem agrupadas, e os avaliadores às variáveis medidas. Ao se considerar 0 conjunto de dados individuais, para cada modelo, os valores dos avaliadores que constituem o 
conjunto de dados multivariados são dados pelas médias dos valores dos avaliadores obtidos para cada animal.

O método de agrupamento utilizado foi o método hierárquico do centroide. Neste método, a distância entre dois grupos é definida como sendo a distância entre os vetores de médias, também chamados de centroides, dos grupos que estão sendo comparados. Segundo Mingoti (2007), é dada, então, pela distância euclidiana ao quadrado entre os centroides dos dois grupos.

Para a determinação do número ótimo de grupos, foi utilizado o índice RMSSTD, que mede a homogeneidade dos agrupamentos; ou seja, quanto menor o RMSSTD, mais homogêneos serão os grupos, condição esta verificada na presença de um grande número de grupos. Este índice, segundo Sharma (1996), é dado por:

$\operatorname{RMSSTD}_{\mathrm{k}}=\sqrt{\frac{\mathrm{SQ}_{1}+\mathrm{SQ}_{2}+\ldots+\mathrm{SQ}_{\mathrm{p}}}{\mathrm{gl}_{1}+\mathrm{gl}_{2}+\ldots+\mathrm{gl}_{\mathrm{p}}}}$,

em que: $\mathrm{SQ}_{\mathrm{j}}=\sum_{\mathrm{i}=1}^{\mathrm{n}}\left(\mathrm{x}_{\mathrm{ij}}-\overline{\mathrm{x}}_{\mathrm{j}}\right)^{2}$ é a soma de quadrado da j-ésima variável calculada, considerando-se as n observações presentes em cada novo cluster k; ou seja, a cada novo cluster, obtém-se um novo valor para o índice em questão.

Após calcular os valores do RMSSTD, é possível construir um gráfico que representa o comportamento desse índice em função do número de grupos. No entanto, essa trajetória não é linear, e o seu ponto de máxima curvatura indica um limiar entre uma fase de decréscimo e uma fase de estabilização. Após este ponto, denominado de ótimo, mesmo aumentando o número de clusters, não se verifica grande declínio nos valores do RMSSTD; ou seja, esse ponto ótimo representará o número ótimo de grupos a partir do qual a estatística mencionada não se altera mais.

Após a obtenção dos grupos (clusters), realizouse a ordenação destes de acordo com as médias calculadas para os avaliadores dos modelos presentes dentro de cada um deles. Como critérios de ordenação, foram considerados os maiores valores de $\mathrm{R}^{2}, \mathrm{R}_{\mathrm{p}}^{2}$ e percentual de convergência, e menores valores de AIC, BIC e MEP.
Os modelos de regressão não linear (Tab. 1) foram ajustados aos dados individuais de crescimento de bovinos por meio do PROC MODEL do SAS/2003. Em relação aos avaliadores da qualidade de ajuste os valores do $\mathrm{R}^{2}$ e da loglike, e do QME foram obtidos diretamente do PROC MODEL. Para a obtenção do MEP e do $\mathrm{R}_{\mathrm{p}}^{2}$, elaborou-se uma adaptação na rotina desse procedimento para remover uma observação a cada ajuste. A porcentagem de convergência diz respeito aos animais cuja convergência foi observada até um número máximo de 1000 iterações.

Após a obtenção dos avaliadores de qualidade para cada modelo, confeccionaram-se então, conjuntos de dados multivariados, os quais foram submetidos à análise de agrupamento no PROC CLUSTER considerando-se o método centroide. Nesta análise, obtiveram-se os valores dos RMSSTD em relação ao número de clusters, a qual gerou o gráfico que disponibilizou a identificação do número ótimo de cluster.

\section{RESULTADOS E DISCUSSÃO}

Na Tab. 2, são apresentadas as estimativas dos parâmetros dos modelos de crescimento ajustados aos dados individuais de bovinos Nelore.

Os modelos Richards, Michaelis-Menten modificado e Weibull (Tab. 2) subestimaram o peso adulto $\left(\beta_{1}\right)$, e os demais estimaram valores para este parâmetro condizentes com os obtidos por diversos pesquisadores (Carrijo e Duarte, 1999; Tedeschi et al., 2000; Garnero et al., 2005; Santoro et al., 2005). Para o parâmetıß̧ 3, que representa a taxa de maturidade, com exceção do Michaelis-Menten modificado e do Weibull, observou-se grande amplitude de variação entre os modelos avaliados - 0,0024 a 0,8653. De acordo com Silva et al. (2004), a variação desse parâmetro pode ser atribuída à alta e negativa correlação entre a taxa de maturidade e o peso adulto, assim os modelos que apresentam baixos valores para $\hat{\beta}_{1}$ apresentam altos valores para $\hat{\beta}_{3}$.

Na Tab. 3, estão apresentados os resultados dos avaliadores da qualidade de ajuste para cada modelo, considerando-se os dados individuais de crescimento. 
Silva et al.

Tabela 2. Estimativas dos parâmetros para os modelos de crescimento de bovinos Nelore, considerandose $o$ ajuste individual

\begin{tabular}{|c|c|c|c|c|}
\hline \multirow[b]{2}{*}{ Modelo } & \multicolumn{4}{|c|}{ Estimativa } \\
\hline & $\hat{\beta}_{1}$ & $\hat{\beta}_{2}$ & $\hat{\beta}_{3}$ & $\hat{\beta}_{4}$ \\
\hline Schnute & 272,6673 & $213,9545^{*}$ & - & 135,3977 \\
\hline Mitscherlich & 495,1949 & $-35,5899$ & 0,002412 & - \\
\hline Richards & 173,9015 & 138,6617 & 0,865346 & $-7,4132$ \\
\hline Gompertz & 383,0079 & 0,8123 & 0,006421 & - \\
\hline Logístico & 343,2097 & 1,7838 & 0,009536 & - \\
\hline Meloun I & 231,4542 & 179,7629 & 0,822111 & - \\
\hline Meloun II & 468,9182 & $-6,0151$ & 0,002625 & - \\
\hline Brody & 495,3454 & 0,9289 & 0,002434 & - \\
\hline von Bertalanffy & 410,5213 & 0,5415 & 0,004652 & - \\
\hline Michaelis-Menten & 553,6187 & 418,8164 & - & - \\
\hline Michaelis-Menten Modificado & 124,8109 & 133251,8 & 424,9587 & $-37,9608$ \\
\hline Weibull & 103,0795 & $-3543,75$ & 312,1085 & $-56,9986$ \\
\hline
\end{tabular}

Tabela 3. Resultados dos avaliadores da qualidade de ajuste para os modelos não lineares, considerandose ajuste individual

\begin{tabular}{|c|c|c|c|c|c|c|c|}
\hline \multicolumn{7}{|c|}{ Avaliadores da qualidade de ajuste } & \multirow[b]{2}{*}{$\mathrm{C} \%$} \\
\hline Modelo & $\mathrm{R}^{2}$ & QME & AIC & BIC & MEP & $\mathrm{R}_{\mathrm{p}}^{2}$ & \\
\hline A - Schnute & 0,7322 & 5283,16 & 78,7738 & 78,6115 & 7234,80 & 0,9150 & 100 \\
\hline B - Mitscherlich & 0,9747 & 372,53 & 61,2488 & 61,0866 & 868,042 & 0,9880 & 79,9517 \\
\hline C - Richards & 0,7189 & 7150,50 & 79,1788 & 78,9624 & 4693,33 & 0,9195 & 8,4541 \\
\hline D - Gompertz & 0,9711 & 457,17 & 63,2762 & 63,1142 & 1067,22 & 0,9859 & 97,1014 \\
\hline E - Logístico & 0,9646 & 579,71 & 65,3381 & 65,1759 & 1304,14 & 0,9835 & 98,7923 \\
\hline F - Meloun I & 0,9672 & 493,63 & 61,7026 & 61,5403 & 1221,06 & 0,9834 & 78,744 \\
\hline H - Meloun II & 0,9710 & 447,82 & 62,3541 & 62,1918 & 6868,61 & 0,8929 & 37,43961 \\
\hline N - Brody & 0,9741 & 381,86 & 61,3352 & 61,1729 & 763,22 & 0,9894 & 80,6763 \\
\hline O - von Bertalanffy & 0,9730 & 420,82 & 62,4535 & 62,2913 & 784,87 & 0,9895 & 95,8937 \\
\hline P - Michaelis-Menten & 0,9661 & 570,06 & 65,3135 & 65,2048 & 862,102 & 0,9877 & 85,2657 \\
\hline Q - Michaelis-Menten M. & 0,7318 & 3976,17 & 65,0316 & 64,8153 & 2576,92 & 0,9537 & 1,93237 \\
\hline R - Weibull & 0,7640 & 2780,31 & 74,6309 & 74,4146 & 5590,13 & 0,9227 & 12,07729 \\
\hline Média & 0,8923 & 1909,47 & 66,7197 & 66,5484 & 2819,537 & 0,9592 & 64,6940 \\
\hline Desvio-padrão & 0,1154 & 2347,22 & 6,7626 & 6,7480 & 2541,761 & 0,0364 & 38,3227 \\
\hline CV\% & 12,93 & 122,92 & 10,13 & 10,14 & 90,14 & 3,80 & 59,23 \\
\hline
\end{tabular}


Todos os avaliadores de qualidade, com exceção do QME, MEP e C\%, apresentaram baixo coeficiente de variação. Resultados semelhantes foram observados por Silveira et al. (2010), em ovinos. A grande amplitude observada para C\% deveu-se ao baixo número de animais que convergiram nos modelos com quatro parâmetros, Richards, Michaelis-Menten modificado e Weibull, mostrando, então, a dificuldade de se trabalhar com modelos mais parametrizados. Os maiores valores do QME também foram observados para os modelos mais parametrizados. O alto CV obtido para o MEP deveu-se ao fato de este avaliador basear-se em remoção sucessiva de observações do conjunto de dados originais para efetuar o ajustamento, portanto ele avalia a capacidade do modelo de se ajustar bem quando se retira o dado (dado faltante). Mas, segundo Nobre et al. (1987), a qualidade dos modelos varia significativamente com a perda de observações, assim a grande variação do MEP deveu-se à perda de qualidade de ajuste do modelo nessas condições. Em relação aos outros avaliadores de qualidade, de forma geral, os modelos da "família" de Richards foram os que apresentaram os melhores avaliadores de qualidade, mostrando a superioridade destes modelos, nesta condição.
Os resultados apresentados na Tab. 3 dos avaliadores da qualidade de ajuste foram utilizados como conjunto de dados multivariados, para a realização da análise de agrupamento. Para a determinação do número ótimo de cluster, foi utilizada a metodologia RMSSTD. Primeiro, foram obtidos os valores do índice RMSSTD (Fig. 1), de acordo com o número de clusters. A partir de seis grupos (Fig. 1), tem-se estabilização da curva exponencial gerada, sendo este o número considerado para formação dos clusters.

Na Tab. 4, constam os clusters com os seus respectivos modelos e médias dos valores dos avaliadores de qualidade do ajuste.

Na ordenação dos clusters, foi dado alto peso ao percentual de convergência (\%C), uma vez que este critério é muito importante em estudos de curvas de crescimento individuais, porque, caso o modelo apresente alta qualidade de ajuste, mas baixo percentual de convergência, o número disponível de animais para seleção seria pequeno. Dessa forma, somente serão utilizados aqueles animais para os quais ocorreu a convergência, cujas estimativas dos parâmetros $\beta_{1}$ e $\beta_{3}$ poderiam ser utilizadas em programas de seleção, para alterar a forma da curva de crescimento.

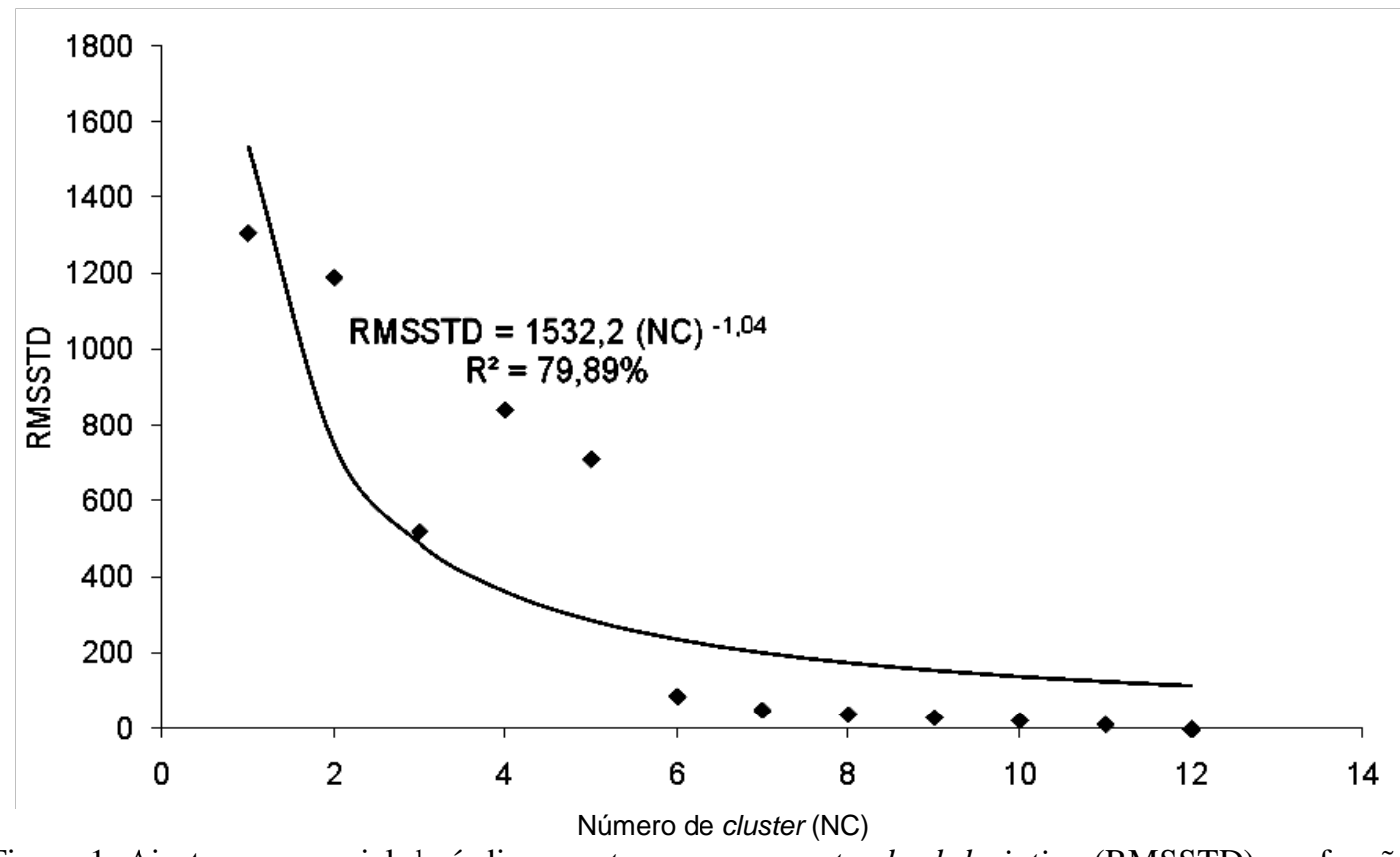

Figura 1. Ajuste exponencial do índice root mean square standard deviation (RMSSTD) em função do número de clusters. 
Silva et al.

Tabela 4. Clusters com respectivos modelos e médias dos avaliadores

\begin{tabular}{|c|c|c|c|c|c|c|}
\hline & Cluster 1 & Cluster 2 & Cluster 4 & Cluster 6 & Cluster 3 & Cluster5 \\
\hline Avaliador & $\begin{array}{c}\text { Modelos } \\
\{\mathrm{N}, \mathrm{O}, \mathrm{B}, \mathrm{E}, \mathrm{F}, \mathrm{P}, \mathrm{D}\}\end{array}$ & $\begin{array}{c}\text { Modelos } \\
\{\mathrm{H}\}\end{array}$ & $\begin{array}{c}\text { Modelos } \\
\{\mathrm{A}\}\end{array}$ & $\begin{array}{c}\text { Modelos } \\
\{\mathrm{Q}\}\end{array}$ & $\begin{array}{c}\text { Modelos } \\
\{\mathrm{R}\}\end{array}$ & $\begin{array}{c}\text { Modelos } \\
\{\mathrm{C}\}\end{array}$ \\
\hline $\mathrm{R}_{\mathrm{aj}}^{2}$ & 0,9701 & 0,971 & 0,7322 & 0,7318 & 0,764 & 0,7189 \\
\hline QME & 467,90 & 447,81 & 5283,16 & 3976,17 & 2780,31 & 7150,55 \\
\hline AIC & 62,9525 & 62,3541 & 78,7738 & 65,0316 & 74,6309 & 79,1788 \\
\hline $\mathrm{BIC}$ & 62,7979 & 62,1918 & 78,6115 & 64,8152 & 74,4146 & 78,9624 \\
\hline MEP & 981,5252 & 6868,61 & 7234,8 & 2576,92 & 5590,13 & 4693,33 \\
\hline $\mathrm{R}_{\mathrm{p}}^{2}$ & 0,9868 & 0,8929 & 0,915 & 0,9537 & 0,9227 & 0,9195 \\
\hline$\% \mathrm{C}$ & 88,0607 & 37,43961 & 100 & 1,9323 & 12,07729 & 8,4541 \\
\hline
\end{tabular}

$\mathrm{R}^{2}$ :coeficiente de determinação; QME: quadrado médio do erro; AIC: valor do critério de Akaike; BIC: valor do critério de informação Bayesiano; MEP: erro quadrático médio de predição; $\mathrm{R}_{\mathrm{p}}^{2}$ : coeficiente de determinação de predição; C\%: porcentagem de convergência. A - Schnute; B - Mitscherlich; C - Richards; D - Gompertz; E Logístico; F - Meloun I; H - Meloun II; N - Brody; O - von Bertalanffy; P - Michaelis-Menten; Q - Michaelis-Menten M.; R - Weibull.

Ainda, de acordo com os resultados da Tab. 4, percebe-se o melhor cluster, o primeiro, pois foi o que apresentou, de forma geral, bons valores para todos os avaliadores considerados simultaneamente. Entre os sete modelos pertencentes a este grupo, pode-se notar que quatro são modelos da "família" Richards. O único desta "família" que não está presente neste grupo é o próprio modelo de Richards, dada a dificuldade computacional envolvida no ajuste deste modelo mais parametrizado. Assim, podese indicar, entre os modelos presentes no primeiro cluster e entre os da "família" Richards, o modelo de Brody como o que melhor se ajustou ao crescimento de bovinos Nelore, por apresentar bons avaliadores de qualidade de ajuste e parâmetros com interpretação biológica. Este resultado é condizente com as observações de Forni (2007), que afirma ser a equação de Brody a mais utilizada para descrever dados de crescimento de bovinos de corte, pela facilidade de convergência dos parâmetros, mesmo na presença de dados faltantes, pelo menor esforço computacional e qualidade de ajuste. Garnero et al. (2005) também sugeriram o modelo de Brody como o mais indicado para descrever o crescimento de animais Nelore, à semelhança dos resultados obtidos por outros autores (Perotto et al., 1992; Oliveira et al., 2000), que também sugeriram a curva de Brody para descrever o crescimento, tanto de bovinos Nelore quanto de animais de outras raças.

\section{CONCLUSÕES}

A metodologia de agrupamento proposta mostrou-se adequada para classificar modelos não lineares, por possibilitar a escolha do melhor modelo para descrever o crescimento de bovinos Nelore. O modelo de Brody, por predizer adequadamente o peso adulto, apresentar bons avaliadores de qualidade de ajuste e alto percentual de convergência, é o recomendado para descrever o crescimento de bovinos Nelore deste estudo.

\section{REFERÊNCIAS BIBLIOGRÁFICAS}

CARRIJO, S.M.; DUARTE, F.A.M. Description and comparison of growth parameters in Chianina and Nelore cattle breeds. Gen. Mol. Biol., v.22, p.187-196, 1999.

CECON, P.R.; SILVA, F.F.; FERREIRA, A. et al. Análise de medidas repetidas na avaliação de clones de café 'Conilon'. Pesq. Agropec. Bras., v.43, p.1171-1176, 2008.

CRUZ, C.D.; REGAZZI, A.J.; CARNEIRO, P.C.S. (Eds). Modelos biométricos aplicados ao melhoramento genético. 3.ed. Viçosa: UFV, 2004. v.1, 480p.

FORNI, S. Análise da curva de crescimento de bovinos da raça Nelore utilizando funções não lineares em análises bayesianas 2007. 75f. Tese (Doutorado) - Universidade Estadual Paulista "Júlio de Mesquita Filho", Jaboticabal, SP. 
GARNERO, A.V.; MARCONDES, C.R.; BEZERRA, L.A.F. et al. Parâmetros genéticos da taxa de maturação e do peso assintótico de fêmeas da raça Nelore. Arq. Bras. Med. Vet. Zootec., v.57, p.652-662, 2005.

MAZZINI, A.R.A.; MUNIZ, J.A.; SILVA, F.F. et al. Curvas de crescimento de novilhos Hereford: heterocedasticidade e resíduos autorregressivos. Cienc. Rural, v.35, p.422-427, 2005.

MENDES, P.N.; MUNIZ, J.A.; SILVA, F.F. et al. Análise da curva de crescimento difásica de fêmeas Hereford por meio da função não linear de Gompertz. Cienc. Anim. Bras., v.10, p. 454461, 2009

MINGOTI, S.A. Análise de dados através de métodos de estatística multivariada: uma abordagem aplicada. Belo Horizonte: UFMG, 2007. 295p.

NOBRE, P.R.C.; ROSA, A.N; SILVA, A.N. et al. Curvas de crescimento de gado Nelore ajustadas para diferentes frequências de pesagens. Pesq. Agropec. Bras., v.22, p. 10271037, 1987.

OLIVEIRA, H.N.; LÔBO, R.B.; PEREIRA, C.S. Comparação de modelos não lineares para descrever o crescimento de fêmeas da raça Guzerá. Pesqui. Agropec. Bras., v.35, p.18431851, 2000.

PEROTTO, D.; CUE, R.I.; LEE, A.J. Comparison of nonlinear functions for describingthe growth curve of three genotypes of dairy cattle. Can. J. Anim. Sci., v.72, p.773-782, 1992.

SANTORO, R.S.; BARBOSA, S.B.P.; BRASIL, L.H.A. et al. Estimativas de parâmetros de curvas de crescimento de bovinos zebu, criados no estado de Pernambuco. Rev. Bras. Zootec., v.34, p.2262-2279, 2005.
SHARMA, S. Applied multivariate techniquies. New York: John Wiley, 1996. 493p.

SILVA, N.A.M., LIMA, R.R., SILVA, F.F.E. et al. Modelo hierárquico Bayesiano aplicado na avaliação genética de curvas de crescimento de bovinos de corte. Arq. Bras. Med. Vet. Zootec., v.62, p.647-654, 2010

SILVA, N.A.M.; AQUINO, L.H.; SILVA, F.F. et al. Curvas de crescimento e influência de fatores não genéticos sobre as taxas de crescimento de bovinos da raça Nelore. Cienc. Agrotecnol., v.28, p.647-654, 2004.

SILVEIRA, F.G. Classificação multivariada de modelos de crescimento para grupos genéticos de ovinos de corte. 2010. 55f. Dissertação (Mestrado) - Universidade Federal de Viçosa, Viçosa, MG.

SILVEIRA, F.G.; SILVA, F.F.; CARNEIRO, P.L.S. et al. Classificação multivariada de modelos de crescimento para grupos genéticos de ovinos de corte. In: REUNIÃO ANUAL DA REGIÃO BRASILEIRA DA SOCIEDADE INTERNACIONAL DE BIOMETRIA, 54., 2009, e SIMPÓSIO DE ESTATÍSTICA APLICADA À EXPERIMENTAÇÃO AGRONÔMICA, 13., 2009, São Carlos. Anais... São Carlos, SP: UFScar, 2009.

TEDESCHI, L.O.; BOIN, C.; NARDON, R.F. et al. Estudo da curva de crescimento de animais da raça Guzerá e seus cruzamentos alimentados a pasto, com e sem suplementação. 1. análise e seleção das funções não lineares Rev. Bras. Zootec., v.29, p.630-637, 2000. 